\title{
High-resolution cryo-electron microscopy on macromolecular complexes and cell organelles
}

\author{
Andreas Hoenger
}

Received: 9 December 2013 / Accepted: 12 December 2013 / Published online: 5 January 2014

(C) The Author(s) 2014. This article is published with open access at Springerlink.com

\begin{abstract}
Cryo-electron microscopy techniques and computational 3-D reconstruction of macromolecular assemblies are tightly linked tools in modern structural biology. This symbiosis has produced vast amounts of detailed information on the structure and function of biological macromolecules. Typically, one of two fundamentally different strategies is used depending on the specimens and their environment. A: 3 -D reconstruction based on repetitive and structurally identical unit cells that allow for averaging, and B: tomographic 3-D reconstructions where tilt-series between approximately \pm 60 and $\pm 70^{\circ}$ at small angular increments are collected from highly complex and flexible structures that are beyond averaging procedures, at least during the first round of 3-D reconstruction. Strategies of group A are averaging-based procedures and collect large number of 2-D projections at different angles that are computationally aligned, averaged together, and back-projected in 3-D space to reach a most complete 3$\mathrm{D}$ dataset with high resolution, today often down to atomic detail. Evidently, success relies on structurally repetitive particles and an aligning procedure that unambiguously determines the angular relationship of all 2-D projections with respect to each other. The alignment procedure of small particles may rely on their packing into a regular array such as a 2-D crystal, an icosahedral (viral) particle, or a helical assembly. Critically important for cryo-methods, each particle will only be exposed once to the electron beam, making these procedures optimal for highest-resolution studies where beam-induced damage is a significant concern. In contrast, tomographic 3-D reconstruction procedures (group B) do not rely on averaging, but collect an entire dataset from the very
\end{abstract}

Handling Editor: David Robinson

A. Hoenger $(\bowtie)$

Department of Molecular, Cellular and Developmental Biology,

University of Colorado at Boulder, Boulder, CO 80309, USA

e-mail: hoenger@colorado.edu same structure of interest. Data acquisition requires collecting a large series of tilted projections at angular increments of $1-$ $2^{\circ}$ or less and a tilt range of $\pm 60^{\circ}$ or more. Accordingly, tomographic data collection exposes its specimens to a large electron dose, which is particularly problematic for frozenhydrated samples. Currently, cryo-electron tomography is a rapidly emerging technology, on one end driven by the newest developments of hardware such as super-stabile microscopy stages as well as the latest generation of direct electron detectors and cameras. On the other end, success also strongly depends on new software developments on all kinds of fronts such as tilt-series alignment and back-projection procedures that are all adapted to the very low-dose and therefore very noisy primary data. Here, we will review the status quo of cryo-electron microscopy and discuss the future of cellular cryo-electron tomography from data collection to data analysis, CTF-correction of tilt-series, post-tomographic subvolume averaging, and 3-D particle classification. We will also discuss the pros and cons of plunge freezing of cellular specimens to vitrified sectioning procedures and their suitability for post-tomographic volume averaging despite multiple artifacts that may distort specimens to some degree.

Keywords Cryo-electron microscopy · Cryo-electron tomography $\cdot 3$-D reconstruction of macromolecular complexes $\cdot$ CTF-correction $\cdot$ Volume-averaging $\cdot 3$-D particle classification

\section{Background}

The evolution of cryo-electron microscopy The nature and quality of electron microscopy image data has changed dramatically with the introduction of cryo-electron microscopy (cryo-EM) and specimen vitrification. Cryo-EM became truly popular in the 1980s. Since the pioneering works of structural 
biologists such as Jacques Dubochet (e.g., see Adrian et al. 1984; Dubochet et al. 1988), Robert Glaser (Taylor and Glaeser 1976), and others, cryo-EM evolved from a highly specialized niche application, employing complicated and sometimes unreliable equipment, to a widely accepted technology that, as of today, produces large amounts of spectacular detailed structural and functional studies on biological macromolecular assemblies to near atomic detail. Cryo-EM relies mostly on the phase contrast produced by the density differences between protein density and the embedding, vitrified buffer. Frozen-hydrated specimens remain unperturbed from staining and/or fixation and maintain atomic detail (Taylor and Glaeser 1976). However, these bright prospects come at a price; vitrified samples produce low contrast, and, unlike chemically fixed, metal-shadowed, or stained preparations, they are very prone to electron beam-induced damage. Nevertheless, propelled by the constantly improving structural data produced by computer-aided, averaging-based 3-D reconstruction methods for macromolecular assemblies such as helical 3-D analysis (DeRosier and Moore 1970), tilt-series reconstructions from 2-D crystalline arrays (Unwin and Henderson 1975), icosahedral viral capsids (Crowther et al. 1970), or single particle (van Heel and Frank 1981; Frank and van Heel 1982) reconstruction methods, electron microscopists soon felt the limitations of the physical resolution limits imposed by negative staining or metal shadowing specimen preparations and demanded molecular fixation methods that enabled atomic details to be obtained. Atomic detail had been first achieved for macromolecular assemblies on 2-D crystalline arrays, and most recently even on pure single particle reconstructions. The history of reaching atomic resolution reads roughly as follows: (a) diffraction data of protein crystals: Taylor and Glaeser 1974 (catalase crystals); (b) 2-D crystals of membrane proteins: Henderson et al., 1990 (bacteriorhodopsin; current world record was achieved with acquaporin- 0 at $1.9 \AA$ (Gonen et al. 2005); (c) 2-D crystalline arrays of soluble proteins: Nogales et al. 1998 (tubulin); (d) helical assemblies: Miyazawa et al. 2003 (acetylcholine receptor reconstituted in lipid tubes) and Yonekura et al. 2003 (bacterial flagella); (e) icosahedral particles: Liu et al. 2010 (adenovirus); (f) single-particle reconstructions: Li et al. 2013 (proteasome).

From noisy cryo-EM 2-D projections to atomic-detail 3-D reconstructions Averaging over hundreds and thousands of identical image elements is an incredibly powerful method to reduce electron microscopy-based image noise, by now often far enough to reveal atomic resolution structural detail. Obviously, averaging can only succeed on structurally identical particles that can be precisely aligned according to their various angular orientations in the recorded 2-D projections. Where applicable, structural variations such as ligand-induced conformational changes have to be recognized and classified accordingly. It is therefore no surprise that 2-D crystals with their strict particle packing regime were among the first macromolecular assemblies from which near-atomic 3-D structural detail was obtained (Taylor and Glaeser 1976; Henderson et al. 1990), while single particle reconstructions only now, and with the help of the latest technology developments on software and hardware (e.g., direct detectors Li et al. 2013; Veesler et al. 2013), seem to promote the breakthrough to near-atomic detail. Thanks to the regular packing of individual units into a highly repetitive structure, with its very practical consequences for Fourier space aided separate analysis of phases and amplitudes, 2-D crystals, helices, and to some extent also icosahedral particles enable the analysis of assemblies of very small $(<40 \mathrm{kDa})$ unit cells. Already the packing procedure into a crystal or a helix acts as a physical filter that either only truly admits identical units, or otherwise provides poor crystallographic packing, which will render these assemblies useless for highresolution analysis (e.g., see Kühlbrandt 1992; Hite et al. 2007). Solubilized single particles such as ribosomes, proteasomes etc. do not assemble into any type of regular array. Hence, electron micrographs typically present them as sets of 2-D projections along very different directions. The most even distribution of projection angles warrants isotropic resolution without a need for recoding tilts. Nevertheless, the signal/noise ratio has to be sufficient to unambiguously reveal the angular relationships between particles, provided they are all identical. If this is not the case, classification has to succeed in identifying distinctly different conformational states (van Heel and Frank 1981; Frank and van Heel 1982; Frank 2009).

\section{3-D cryo-electron microscopy on large, flexible structures The} spectacular success of 3-D reconstructions by cryo-EM and averaging procedures should not make us forget the fact that the 3-D architecture of the vast majority of biological structures cannot be subjected to averaging methods. The larger a macromolecular assembly or cell organelle is, the higher the probability of intrinsic flexibility, and the lower are the chances of structural identity between multiple copies (e.g., Figs. 1, 2, and 3). If averaging and/or alignment procedures of particles fail, the only way to obtain 3-D data is cryo-electron tomography (cryo-ET; Medalia et al. 2002; Beck et al. 2004, reviewed in Steven and Aebi 2003; Lucic et al. 2005). Today, high-resolution cryo-ET is probably the most active and fastest emerging discipline in structural cell biology as it can be applied to large macromolecular assemblies, cellular organelles, or even entire tissues. By omitting the need for any kind of averaging procedures, at least during the initial round of 3-D reconstruction, tomography can be applied to any specimen that is thin enough to get an electron beam through. Intact bacterial cells such as Caulobacter crescentus (e.g., see Briegel et al. 2006, 2009; multiple examples reviewed in Gan and Jensen 2012), or flat areas of eukaryotic cells (e.g., fibroblast peripheries; Dictyostelium: Medalia et al. 2002) may be suitable for direct imaging. All other cellular specimens have 

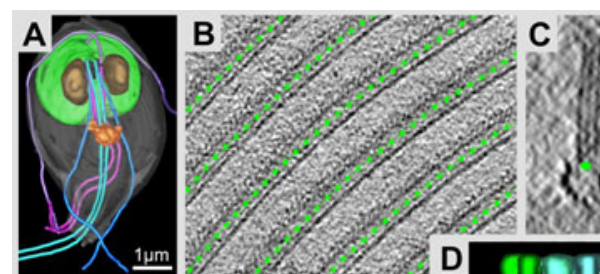

(n) 3

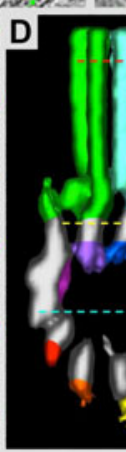

Fig. 1 Subvolume averaging from tomograms of plunge-frozen, regular arrays within the unique Giardia lamblia cytoskeleton. a Microtomebased block-face scanning-EM imaging of a Giardia trophozoite reveals sufficient resolution to identify flagella, nuclei $(N)$, the median body $(M B)$, and ventral disc $(V D)$. These arrays constitute excellent test grounds for our labeling experiments as they allow averaging of subvolume elements and calculating difference maps for a precise detection of clonable labels. a Tomographic 20-nm thick X-Y slice of the Giardia ventral disc (green organelle in the Giardia cell shown in (c, d) at the level of the microtubule arrays. c Tomographic 20-nm thick X-Z slice of the Giardia ventral disc showing microtubules and associated microribbons end-on. d, e 3-D reconstruction of the microtubule-microribbon complex of the Giardia ventral disc. d End-on view (left panel towards the microtubule plus-end) and side view (right panel) of a grand average over 4,700 individual tomographic subvolumes. MT protofilaments are numbered, starting at the position of the seam. The largest associated densities are called side-arms (green). Currently, we do not know how many individual protein domains are within this structure. e Panels top to bottom show cross-sections at corresponding position in the 3-D map marked by lines of the following color: red (microribbons), yellow (upper microtubule protofilaments), and cyan (microtubule lumen and side arm densities). The cross-section through the microribbons reveals a distinct 16-nm repeat, corresponding to two consecutive $\alpha \beta$-tubulin dimers along a protofilament. Side-arms repeat in register with the tubulin dimer repeat. The 3-D map still suffers from a missing cone of data (see also Fig. 4), demonstrated by the clear separation of protofilaments horizontally, but not vertically (for further details see Schwartz et al. 2012)
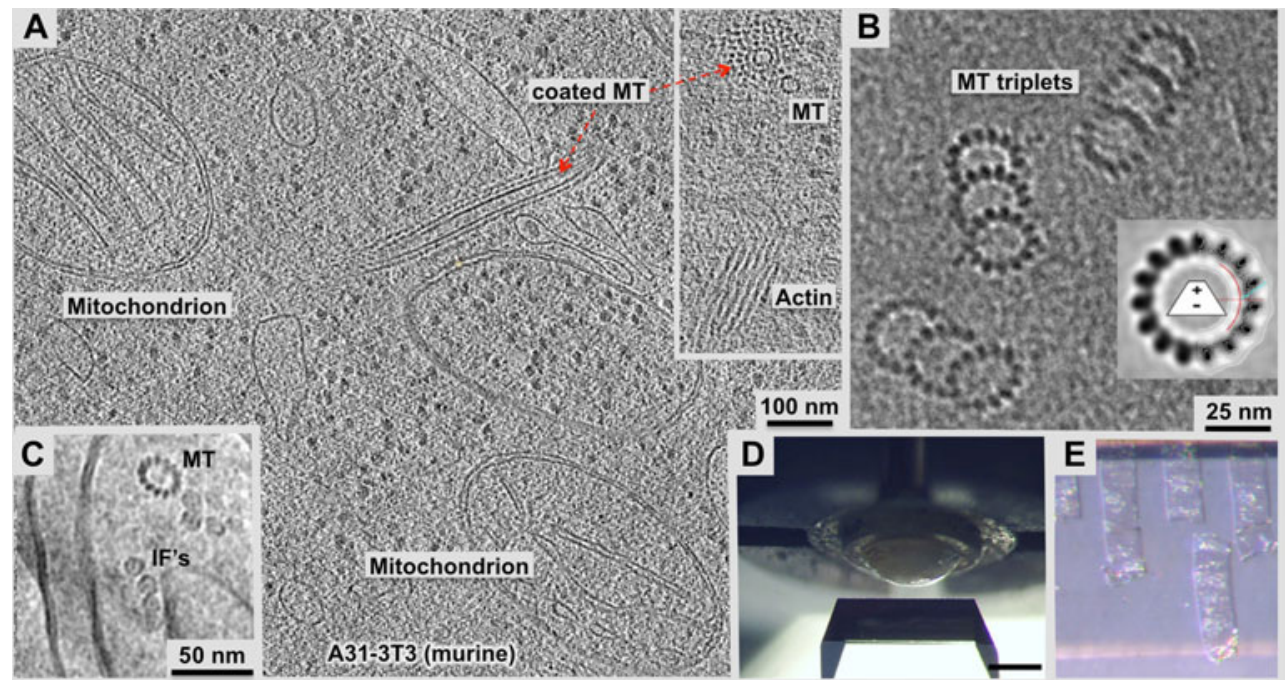

Fig. 2 3-D analysis of large cells and tissues: Intracellular molecular details preserved in vitrified sections. a, b $70 \mathrm{~nm}$, frozen-hydrated cryosection observed in a high-pressure frozen A31-3 T3 cell. a Approximately 10 -nm thick tomographic slice that shows mitochondria, ribosomes, endosomes, and an actin-coated microtubule. Inset: end-on view of such a coated microtubule, and side view of an actin bundle. b An important advantage of CEMOVIS is illustrated in these triplet microtubules of the centriole where the technique allows for a direct determination of the microtubule polarity due to the characteristic slew of the protofilaments in end-on views (compare to helical averaging of a set of microtubules in the inset). Here, we are looking from the minus towards the plus end. c
Cross-sections of a microtubule next to vimentin intermediate filaments $(I F s)$ in a vitrified section of $\mathrm{CHO}$ cells. All of the vimentin filaments show a clear central density, which is different from data obtained on in vitro polymerized vimentin IFs (see Goldie et al. 2007). $\mathbf{d}$ Frozen cell pellet in a dome-shaped carrier approaching the cryo-diamond knife for trimming, $\mathbf{e}$ $100-\mu \mathrm{m}$ wide, $50-\mathrm{nm}$ thick vitrified sections are shown on the surface of a $45^{\circ}$ cutting cryo-diamond knife ready to be collected on a carbon-coated, copper grid. Cryo-sections have a transparent to silver, shiny look. Unlike plastic sections, vitrified sections cannot be floated on a water surface for obvious reasons. Hence, they have to be picked up by a micromanipulator and transferred to an EM grid 

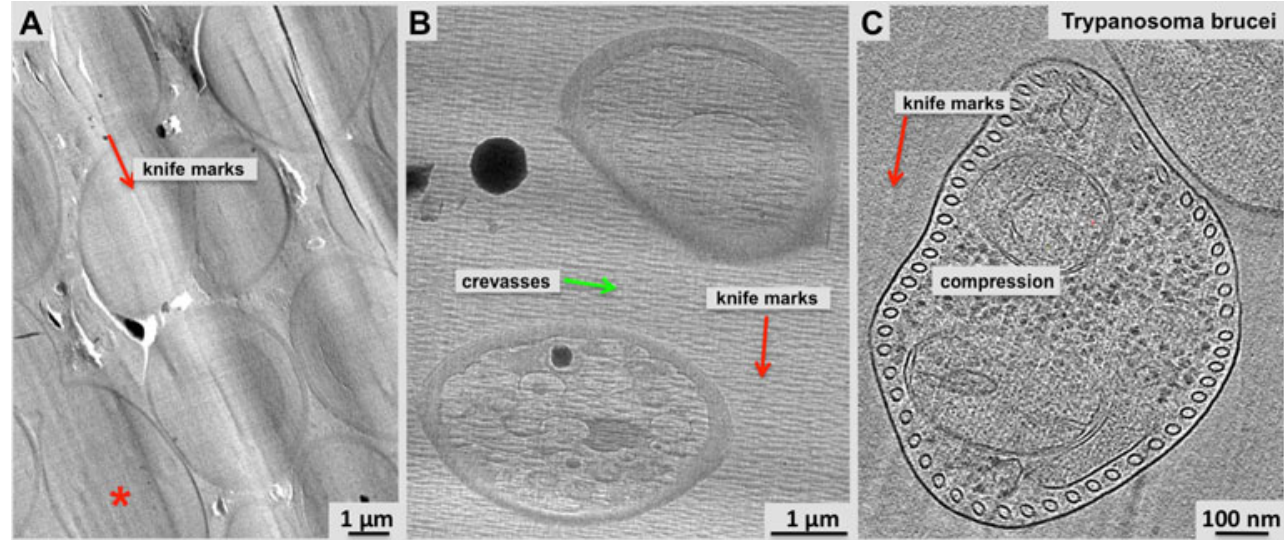

Fig. 3 The vitrified section process affects the specimen geometry in various ways: Damage by the cutting knife such as compression or crevasses and its effect to macromolecular structures has to be carefully analyzed before volume averaging procedures can be applied. Largescale distortions and compression along the cutting directions (red arrows) affect membrane organelles, including microtubules cross-section and shape (MT in a, c). a 2-D projection micrograph through a vitrified section of $3 \mathrm{~T} 3$ cell showing multiple membrane vesicles and some microtubules towards the center. Most organelles appear

to be treated by vitrified sectioning (e.g., see McDowall et al. 1983; Al-Amoudi et al. 2004a; Dubochet et al. 2007; Bouchet-Marquis and Hoenger 2011), or by focused ionbeam milling in a dual-beam cryo-scanning electron microscope (reviewed in Lucic et al. 2013).

Tilt-series data collection and data processing in electron tomography is much more demanding with respect to computing power and data storage than diffraction-based averaging methods where 2-D and 3-D datasets often can be reduced to rather small sets of numerical structure factors. Individual tomograms (conventional or cryo-ET) easily exceed a gigabyte (GB) in size, and computationally combined super-montages (Mastronarde et al. 2008; currently still a domain of plastic-section tomography) may be as large as 10-20 GB and beyond. Compared to conventional plastic section tomography where grids are often pre-exposed to the beam to avoid shrinking during data collection, cryo-ET adds a complication to the process due to its dramatic sensitivity to the electron beam. The total dose specimens are exposed to during tilt-series acquisition has to be carefully considered and kept below values that would destroy projections towards the end of a tilt series. The resulting individual low-dose images are extremely noisy and may often lack the so-called fiducial markers due to the difficulties when handling vitrified specimens that may complicate the proper alignment of individual tilted projections for the 3-D backprojection process. Recent progress in microscope stage designs as well as camera hardware (direct electron detectors; Jin et al. 2008; McMullan et al. 2009a; Campbell et al. 2012) will be very beneficial for progress in cryo-ET and will be discussed below. compressed along the cutting direction. b 2-D projection micrograph of a vitrified section through $S$. pombe cells revealing the typical distortions encountered during vitrified sectioning such as knife marks and crevasses. c 21-nm tomographic slice through a cross-section of a Trypanosoma brucei cell reveals the spectacular microtubule-based cytoskeleton of this single-cell organism around the edge. Most of the microtubules appear squeezed roughly along the direction of the knife marks, though the compression is not uniform (see also Höög et al. 2012)

\section{Exploiting the full potential of cryo-ET 3-D analysis: processing the raw data}

Knowing and understanding the challenges that are facing us is typically the first step to success. Sophisticated technologies such as cryo-ET always come with their unique sets of issues and demands. TEM-based cryo-electron tomography of frozen-hydrated samples features several challenges for data acquisition, data analysis, and data interpretation that all require careful consideration. Regarding imaging and data acquisition, frozen-hydrated specimens - due to low intrinsic contrast - are recorded at large underfocus values, typically between -2 and $-6 \mu \mathrm{m}$, which creates images, mostly from phase contrast that are strongly affected by the contrast transfer function (CTF). The CTF is particularly demanding to assess on highly tilted specimens where focus values gradually vary perpendicular to the tilt axis. In addition, cryoholders and the geometrical nature of a TEM specimen stage limit the range for tilt series to maximally $\pm 70^{\circ}$, which creates a missing wedge of data (see Figs. 1 and 4). With a maximum tilt of $\pm 60^{\circ}$, the missing wedge reduces resolution along the projection direction and tilt axis by $50 \%$ (Fig. $4 \mathrm{~b}$ ) and affects further 3-D analysis procedures such as alignments (in particular for fiducial-free alignment; Winkler and Taylor 2006; Castano-Diez et al. 2007) and classification of subtomogram volumes picked for averaging (Förster et al. 2008; Winkler et al. 2009; Heumann et al. 2011; Yu and Frangakis 2011). However, these kinds of distortions apply to any kind of cryo-sample and each element in a cryo-micrograph or cryo-tomogram. Most of them are quite well understood and several correction procedures have been published from our 


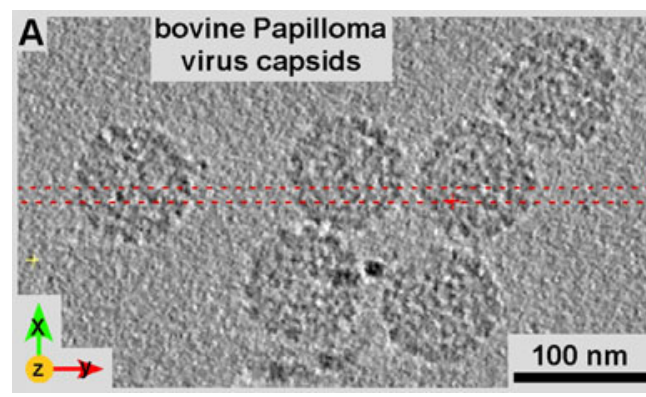

Fig. 4 Effects of the missing wedge on the image quality in tomograms illustrated on bovine Papilloma virus capsids. Tomograms are unisotropically resolved, lacking resolution along the $z$-axis (in the case of a missing cone) and $z$-axis and along the tilt-axis (missing wedge). While the XY-view (a) typically look highly resolved, the anisotropy of image resolution in tomograms becomes very obvious when viewed along the $x$ or $y$-axis (b). Typically, the $z$-axis becomes stretched and has

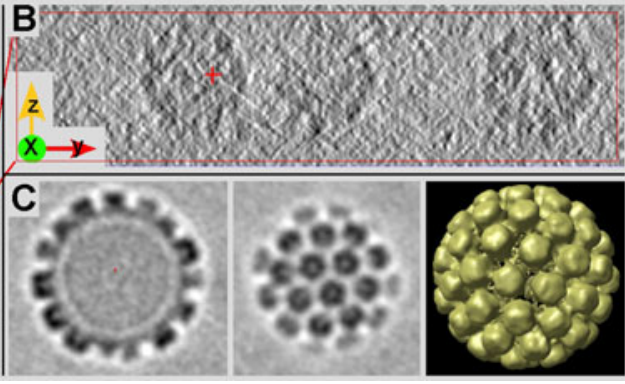

less resolution. Averaging multiple, structurally identical particles viewed under various angles may resolve that problem and restore the resolution equally in all directions. c 3-D structure of BVP at $\sim 2.5-\mathrm{nm}$ resolution obtained by volume averaging from the data shown in $\mathbf{a}$ and $\mathbf{b}$. After averaging together particles with different orientations in the tomogram, the resolution is now isotropic

been made available in our own IMOD software package and eTomo interface and is now used routinely. It can be successfully used with vitrified sections, where relatively few large patches are tracked, as well as with stained plastic sections, where many smaller patches can be tracked. (Mastronarde 2008), are routinely applied.

Fiducial-less alignments of tilted projections is currently the standard for cryo-ET Whenever possible, tilt series are aligned to each other with the help of electron-dense fiducial markers, typically gold particles from $5-15 \mathrm{~nm}$ in diameter that can be easily added to grids and plastic-thin sections that are handled at ambient conditions (room temperature, no vacuum; Mastronarde 2007). In contrast, fiducial markers are often difficult to incorporate into frozen-hydrated samples (see Masich et al. 2006; Gruska et al. 2008). The Giardia lamblia ventral disc structure presented in Fig. 1c, as well as the tomograms of Figs. 2a and 3c, are all products of fiducialless alignments. Hence, successful alignment procedures for tilt series without fiducial markers are very important for most kinds of plunge-frozen specimens and in particular for vitrified sections. Among the first to tackle this problem was the group of Winkler and Taylor (2006), but their method that relied on tracking image features through the entire set of tilted projections had some limitations. Much greater success was achieved with a novel approach developed by Castano-Diez et al. (2007, 2010): correlating multiple overlapping patches of image through the tilt series and treating the tracked positions like a fiducial model. Because alignment data are available at multiple positions, it is possible to solve for the tilt axis angle, for specimen shrinkage, and even for refined tilt angles in some cases. However, since image patches rather than specific features are tracked, the tracking may not follow a consistent position through the whole series, just as an overall cross-correlation may not. The effects of this positional variation can be ameliorated by subdividing each track into overlapping segments (Castano-Diez et al. 2010). This method has

\section{Exploiting the full potential of cryo-ET 3-D analysis: volume averaging of tomographic data}

High-resolution cryo-ET We like to speak of "high-resolution cryo-ET." However, compared to the typical averaging-based methods described above, the meaning of "high" has to be seen in relative terms; for averaging-based 3-D reconstructions today, high-resolution means near-atomic detail. High-resolution cryo-ET datasets today, after producing 2-3-nm detail for some time, now start to break through the $2-\mathrm{nm}$ resolution barrier $(<1 \mathrm{~nm}$ in some cases; see Fig. 5 and Schur et al. 2013). Beyond 2-3-nm resolution, unambiguously interpretable structures are typically only resolved by post-tomographic volume averaging (e.g., see Nicastro et al. 2006; Cope et al. 2010; Schwartz et al. 2012; Kuybeda et al. 2013; Schur et al. 2013). All other cryo-tomography reconstruction data that have not been averaged in any way, unambiguously interpretable structural data remains in a 3-4-nm range.

Post-tomographic sub-volume averaging fully exploits the resolution of cryo-ET Having praised conventional and cryo-ET for their independence from averaging procedures does not mean there is no post-tomographic averaging possible with tomographic 3-D data. Once a tomogram is calculated, there may be numerous objects in such a 3-D dataset that can be averaged in forms of 3-D sub-volumes, provided there are identical particles to be found (e.g., ribosomes; Pierson et al. 2011; Figs. 1 and 4). Aligning 3-D volumes along all three axes increases the demand for fast computers and 
algorithms, but features substantial advantages over picking and classifying single particles as 2-D projections. As for 2-D projections, averaging structurally identical 3-D volumes improves the signal/noise ratio in these datasets, in an ideal world by about the square root of numbers of particles. However, there is a crucial difference between averaging and 3-D reconstructing 2-D projections and averaging readily available 3 -D datasets. Their alignment is computationally much more demanding but reconstructed 3-D volumes already contain much more information than 2-D projections where superimposed densities require careful de-convolution. Subvolume averaging not only improves resolution but it may be a way to overcome intrinsic tomography-related issues such as filling in missing wedge or cone data by averaging over particles in different orientation (Fig. 1) or with high internal symmetry (Fig. 4).

Classification and variance maps As for the regular averaging process described above, 3-D volumes significantly facilitate the alignment process with the benefit of reducing the noise level of cryo-ET data, but also allowing for assessing possible heterogeneity of the particles being averaged and for clustering them into separate classes according to distinct conformational states and/or composition with multiple components (Förster et al. 2008; Heumann et al. 2011; Frank et al. 2012; recently reviewed in Briggs 2013). However, the intrinsic missing tomographic data caused by missing wedges or cones, as well as low signal-to-noise ratio, make classification problematic. In our lab, we have developed a new method for clustering in the face of these difficulties that aims at estimating the difference between observed and expected particles, taking the potential effects of missing wedge data into account. Differences between expected and observed sub-volumes, which we term "wedge-masked differences" (WMDs), can then be analyzed using standard statistical methods (Van Heel and Frank 1981; Frank and van Heel 1982). We developed programs using WMDs for computation of a corrected variance map and for classification based on principal components analysis followed by K-means clustering (Heumann et al. 2011).

Several labs, including our own, have invested substantial effort into the development of volume averaging procedures (e.g., our PEET software package: applications in Nicastro et al. 2006; Cope et al. 2010; Schwartz et al. 2012), classification protocols (Heumann et al. 2011; Frank et al. 2012), as well as corrections for the contrast transfer function (Xiong et al. 2009). Today, volume averaging and classification of 3$\mathrm{D}$ particles is a hot topic in the cellular tomography community (e.g., see Kuybeda et al. 2013; Frank et al. 2012; Yu and Frangakis 2011; Kudryashev et al. 2012; Bartesaghi et al. 2012) and our lab and others continue to be at the forefront of this developing technology.

\section{Exploring the 3-D structure of intact cells by cryo-ET}

Cryo-electron tomography of vitrified sections Until recently, cellular EM was synonymous with rapid-freezing and freezesubstitution fixation (RF-FSF) protocols followed by plastic embedding for many biologists (Carlemalm et al. 1985; Kellenberger 1991; McIntosh et al. 2005). In fact, RF-FSF is still the method of choice for investigations into the cellular architecture and the organization of organelles in the cytosol (Figs. 2 and 3; Marsh et al. 2004; Pelletier et al. 2006; O’Toole et al. 2007; Ferguson et al. 2007; Höög et al. 2007; see also article by Kent McDonald, this issue). Like the "pure" cryomethods, RF-FSF employs an initial vitrification step with a plunge or high-pressure freezer, and as such, it constitutes a reliable method for observing cellular data to about $5-\mathrm{nm}$ resolution (Studer et al. 1989; McDonald and Morphew 1993).

The protocols for vitrified sectioning omit the freezesubstitution and plastic-embedding step and cut sections straight from a block of a frozen-hydrated specimen. Today, the procedure is often referred to as cryo-EM on vitrified sections (CEMOVIS; Al-Amoudi et al. 2004a), and has actually been around for some time (Christensen 1971; McDowall et al. 1983; Hsieh et al. 2002; Al-Amoudi et al. 2004a, b; Dubochet et al. 2007) so that this acronym seems to be part of a Swiss insider joke. Although the first vitrified sections were produced as early as 1971 (Christensen 1971), cryo-electron tomography on vitrified sectioning was significantly facilitated only after substantial technical developments on cryo-EM-related tools such as cryoholders, plunge-freezers, high-pressure freezers, ultra-sensitive detectors, and last but not least by the availability of fast, efficient computers and software became available (e.g., see Patwardhan et al. 2012). Even today, the technology requires a lot of manual skills and is only used by few labs. Vitrified sectioning reveals images of cellular organelles and macromolecular assemblies in a frozen-hydrated state, thereby omitting both contrast-enhancing staining solutions as well as chemical fixatives such as glutaraldehyde. The most important advantage resulting from cryo-immobilization of biological samples is a superb structural preservation under most native buffer conditions, which closely resembles the living state of a cell and maintains the structure of macromolecules down to atomic detail (Dubochet et al. 1988; Baumeister and Steven 2000; McIntosh 2001; Steven and Aebi 2003). Accordingly, the expectations for structural preservation and achievable image resolution are high, but a realistic assessment of the method's advantages and limitations has to be done before investing in that technology (e.g., see Dubochet et al. 2007; Pierson et al. 2010; Bouchet-Marquis and Hoenger 2011).

Vitrified sectioning, if exploited for its true potential does not compete with, but is very complementary to plasticembedding and conventional ET. CEMOVIS clearly has the capacity to deliver a different type of information such as more detail from intracellular molecular structures rather than 
large overviews. Thus, the most important aspect with regard to vitrified sections is the full exploitation of the image resolution since molecular structures are at no time exposed to staining solutions or chemical fixatives. Combined cryoelectron tomography and single-particle 3-D reconstruction protocols (Kuybeda et al. 2013), or sub-volume averaging procedures (Schur et al. 2013), may provide sub-nanometer detail. However, the question remains as to how good are these protocols when transferred to cellular specimens that have been subjected to vitrified sectioning. On a first inspection, the structural information that is directly interpretable in vitrified sections may not be that much richer in detail than from plastic-embedded sections, although this may vary with the object. Typically, the full potential of vitrified sections can only be exploited by additional averaging-based processing methods (see Figs. 1 and 4). A plain 2-D image of a vitreous section that has been recorded under low-dose conditions ( $\sim 1-$ 5 electrons per $\AA^{2}$ ) may theoretically hold near atomic detail, but low contrast and the super-positioning of cellular densities in a 50-80-nm thick section through dense cellular material makes it difficult to analyze small structures. Furthermore, a cryo-tomogram of a vitrified section is a composite of multiple projections from a tilt series (typically $100-140$ at $1-2^{\circ}$ intervals) where the final resolution in the tomogram is very dependent on the successful reconstruction and the stability of the specimen in the electron beam. Even in near-perfect tomograms, the signal/noise ratio is still rather low (Fig. 5).

Compression, crevasses, and other problems Currently, probably the toughest challenge on vitrified sections comes from distortions that are implemented during the sectioning process (Fig. 3; see Al-Amoudi et al. 2005; Dubochet et al. 2007; Han et al. 2008; Bouchet-Marquis and Hoenger 2011). Vitrified sections are cut through relatively brittle ice, leaving knife marks (Fig. 3a-c) and crevasses (Fig. 3b), particularly at the side facing away from the knife surface. Other common sectioning-induced distortions are compressions (Fig. 3c) that affect different structures in a section with very different severities. However, despite these conditions, vitrified sections preserve high-resolution structures very well (Sader et al. 2009). With regard to exploiting high-resolution data from vitrified sections, it is these compressions in particular that require more careful study. The remaining question is how much is volume averaging of macro-molecular structures affected by compressions, and which kind of molecular structures can still be analyzed by averaging procedures.

\section{Clonable high-electron dense labels for vitrified specimens}

How to navigate the complexity of proteinaceous densities in vitrified cellular specimen This final chapter touches an issue that is not only relevant for cryo-EM or cryo-ET, but concerns all cellular electron microscopy in general. Localizing a particular structure of interest within the crowded environment of a cytosol can be very challenging. Recognizing the components of interest at in vitro condition seems straightforward, but even in vitro labels may be very helpful (e.g., see Cope et al. 2013). Unambiguously, identifying a macromolecular assembly within the crowded environment of the cytosol without any type of labeling can be very difficult, if not impossible. Within intact cells, we may easily be able to identify unambiguously microtubules or ribosomes (e.g., see Figs. 1, 2, and 3), but most other structures remain hidden away by the multitude of dense complexes within the cytosol.

Plastic-based thin-sections can be handled at room temperature and therefore may be treated with gold-labeled antibody solutions to localize surface-exposed epitopes on sections (e.g., see Tokuyasu 1980). However, the situation is very

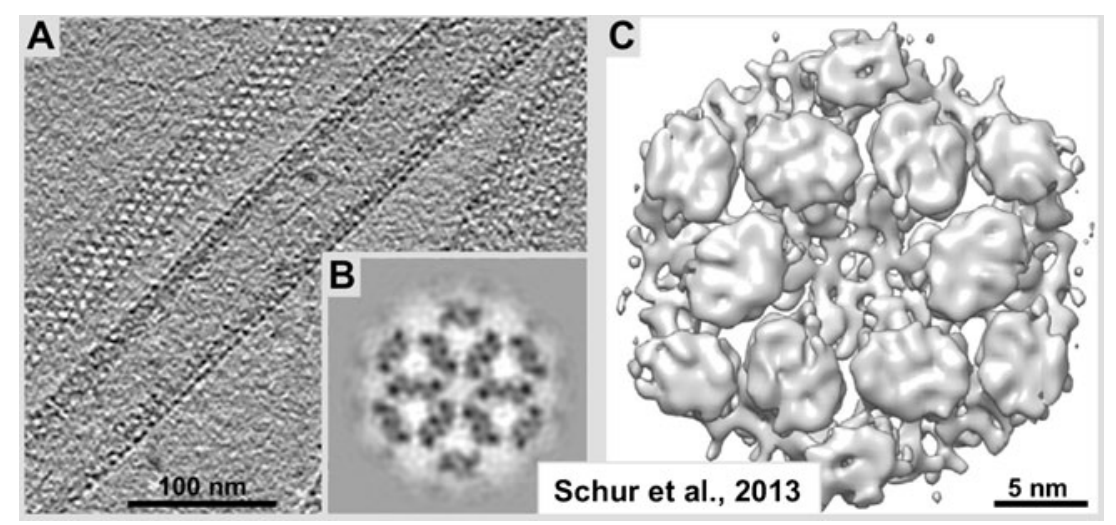

Fig. 5 Sub-tomogram averaging reconstruction from tubes of M-PMV (Mason-Pfizer monkey virus) DPro CANC protein (shown in a). b Graylevel, and $\mathbf{c}$ iso-surface representations hexameric DPro CANC protein unit cells. For details see Schur et al. 2013. Currently, this reconstruction is among the best-resolved datasets obtained by sub-tomogram averaging.
At $8.5 \AA$ resolution, this 3-D dataset from the lab of John Briggs at the EMBL-Heidelberg exceeds all previous expectations and opens the avenue for secondary element studies by cryo-ET. Adapted from Schur et al. 2013, Figs. 2a and 4a, b, with permission from Drs. J. Briggs and F. Schur 
different with vitrified specimens. The most important differences between a frozen hydrated, or vitrified specimen, and one that is either air-dried or plastic-embedded are as follows: (a) once frozen, a vitrified specimen has to be kept below the devitrification temperature $\left(133 \mathrm{~K}\right.$, or $\left.-140{ }^{\circ} \mathrm{C}\right)$ of water at all times, and (b) cannot be exposed to ambient conditions for more than a few seconds (e.g., during the grid-transfer into the microscope, otherwise the specimen, acting as a cold trap, would be heavily contaminated by ice from humid air. (c) Vitrified specimens are extremely sensitive to the electron beam. That not only calls for expensive equipment such as cryo-holders and high-vacuum stages, but it also prevents essentially every kind of after-freezing treatment with substances that are warmer than $133 \mathrm{~K}$. Hence, there are no protocols for applying antibodies or any other liquids, including solutions with fiducial gold markers, to a vitrified specimen. Hence, all protein tagging has to be done before freezing the specimen, preferably with clonable tags that can be made visible in the electron microscope. Such tags include clonable metal-clustering proteins or peptides (e.g., see metallothionein Fig. 6 and Mercogliano and DeRosier 2006; A3-peptide Slocik et al. 2005), as well as aptamer-based strategies (Stanlis and McIntosh 2003; Feldheim and Eaton 2007), such as linking them to boranephosphate DNA segments or other metal precipitating structures (Roy et al. 2012, 2013).

My own efforts to develop clonable labels dates back to approximately 2002 while I was still working at the EMBL in Heidelberg Germany (Wendt et al. 2002; Skiniotis et al. 2003). Eventually, clonable labels for electron microscopy will fulfill a similar function to clonable labels for fluorescence light microscopy, such as GFP, mCherry, etc. They have the advantage of being directly linked to the protein of interest, providing increased spatial resolution. However, while the tags now work rather well in in vitro situations (Bouchet-Marquis et al. 2012), their applicability within living cells has to be proven first. Clonable labels that are relevant for electron microscopy studies may be placed in three classes as follows: (a) simple protein domains such as an SH3 domain (e.g., see Wendt et al. 2002; Skiniotis et al. 2003), (b) peptides that reduce metal ions into electron dense clusters (e.g., the gold-clustering A3-peptide; Slocik et al. 2005), or (c) protein domains that chelate metal ions with reactive elements such as thiol groups of cysteine (e.g., metallothionein; Mercogliano and DeRosier 2006). The most important advantage of metal reducers or chelators is that because of their high electron density, these labels stand out over the average protein density within a cell or organelle. Regular protein domains such as an SH3 domain were shown to be very useful in vitro and on specimens that allowed averaging and statistical difference mapping. However, where protein tags must be visible without computational help, the metal cluster tags are obviously the tags to choose.

The downside of clonable labels in general is that the label may interfere with the function of the labeled protein, or prevents its correct localization in the cell, which has been found in some cases for fluorescent labels such as GFP (Werner et al. 2009). While the first clonable labels we investigated were simply small proteinaceous particles such as an SH3 domain (Wendt et al. 2002; Skiniotis et al. 2003), the more recent ones are either metallothionein (MTH; Mercogliano and DeRosier 2006) or metal-clustering peptides (e.g., the gold-clustering A3-peptide; Slocik et al. 2005). Although there have been a few reports on successful attempts to use MTH as an intracellular label (Diestra et al. 2009a, b; Risco et al. 2012), in our experience, an intracellular
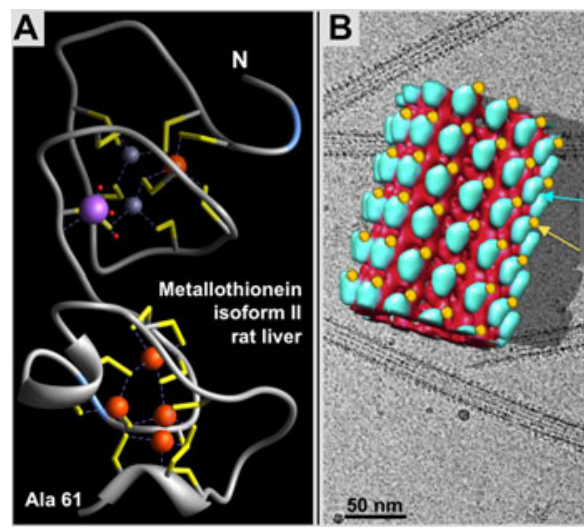

Fig. 6 Clonable, gold-clustering labels. Metallothionein (MTH) complexed with gold constitutes a clonable high-density marker for electron microscopy (Mercogliano and DeRosier 2006). a Atomic-detail NMR structure of rat liver MTH (isoform II) at 2.0- $\AA$ resolution (Braun et al. 1992). The structure was solved with five Cadmium ions (orange), two Zinc ions (small purple spheres) and one Sodium ion (pink) clustered within the molecule. MTH contains 20 cysteine residues (yellow sticks) that achieve the clustering of metal ions by chelation with their cysteinethiol groups that can accumulate to 20 and more gold atoms within the
Microtubules dec. with Kinesin Eg5-MTH (Zn)
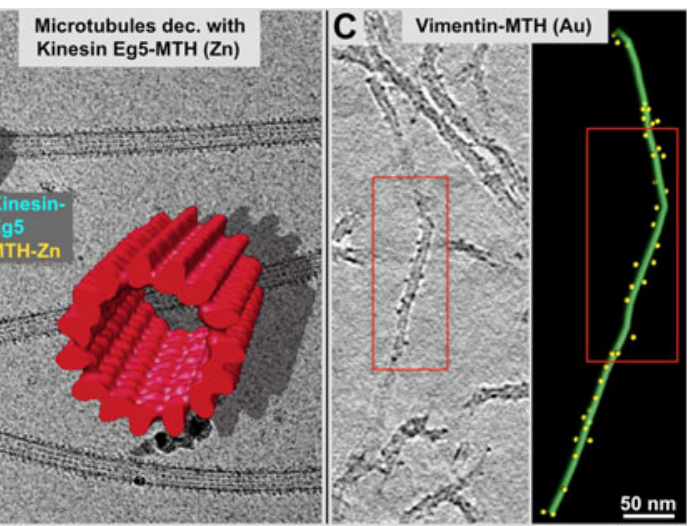

crystal structure forming easily detectable high-density cluster b Metallothionein-Eg5 microtubule complexes with a helically averaged 3-D reconstruction highlighting the MTH-zinc densities (yellow) and their position relative to $\mathrm{Eg} 5$ (blue). A helical average of an undecorated microtubule is shown in red. $\mathbf{c}$ Desmin-MTH chimera polymerized into intermediate filaments present the MTH-gold clusters on their surface. Left panel 3-D model of the center filament in C (compare red frames). See also Bouchet-Marquis et al. 2012 
application of MTH or the A3 peptide, followed by loading them with gold to generate a visible high-electron density cluster, has proved very challenging. While proteins tagged with a small peptide or a small protein domain may still function properly, once the tag is loaded with metal, there is an increased chance that the physiology of the protein of interest will be affected due to the large mass of the tag. In our own lab, we found almost by accident that MTH clusters zinc very well because we supplemented media with excess zinc (less toxic than gold) to prevent MTH from sequestering essential metals from the cytosol. We succeeded with MTH and A3 peptide applications for in vitro experiments (see Fig. 4; BouchetMarquis et al. 2012), but so far we have been able to load intracellular MTH only with zinc. Zinc, however, has a significantly lower electron scattering potential than gold and is easier to detect by electron energy loss spectroscopy (EELS) than gold because unlike gold, zinc produces a sharp energy absorption edge $(-18$ and $-1,020 \mathrm{eV})$. Nevertheless, EELS is not a current option for vitrified specimens due to the large amount of electron scattering required to generate a useful signal.

\section{Recent hardware developments for cryo-EM and cryo-ET}

The last few years have seen a dramatic change in cryo-EM and cryo-ET data collection through the introduction of the direct electron detector cameras that work with CMOS chips. Similar to regular photography, CMOS chips mark a quantum leap for cryo-EM data acquisition. The direct electron detector technology omits the scintillator and fiber optics, which lowered image quality through their significant point-spread function as well as noise generation by back-scattered electrons. Back-scatter can still be a problem in direct electron detector cameras, but it can be largely overcome by back-thinning the support layer (McMullan et al. 2009b). New high-speed subframe readout procedures allow for some image corrections such as drift already during data acquisition (Jin et al. 2008; Campbell et al. 2012). If the chip is read out fast enough and the rate of incoming electrons is low enough, individual primary electrons can be detected and their exact location estimated, thus eliminating both signal spreading and packet variability as limiting factors (McMullan et al. 2009a). Gatan Inc. has implemented such electron counting in the K2 Summit camera. In addition, the K2 Summit camera has a super-resolution mode, in which the estimates of electron location are used to form an image with pixels half as big as the physical ones. Hence, our expectations from these new cameras are high.

Other significant improvements, especially for cryo-ET, were the development of cartridge-based cryo-stages that disconnected the specimens from the outside. These stages were implemented first in the FEI Polara, and JEOL-3100FCC microscopes, and further perfected with the FEI Titan-Krios microscopes where the cartridge system was combined with an automated multi-specimen autoloader. The significantly better stability of all these stages during tilt-series data acquisition is undisputed. Furthermore, while extremely successful for highest-resolution EM on non-biological material, the benefits of $\mathrm{Cs}$ and $\mathrm{Cc}$ correctors as well as the constant-current lenses, introduced with the FEI Titan product line, have yet to be carefully evaluated for cryo-ET on biological specimens. But they may be the technologies that will further shape the bright future of cryo-ET on cells and macromolecular structures.

Acknowledgements Credits for unpublished images in figures 2, 3 and 6B: Cedric Bouchet Marquis, figure 4: Mary Morphew, figure 6C: Robert Kirmse

Open Access This article is distributed under the terms of the Creative Commons Attribution License which permits any use, distribution, and reproduction in any medium, provided the original author(s) and the source are credited.

\section{References}

Adrian M, Dubochet J, Lepault J, McDowall AW (1984) Cryo-electron microscopy of viruses. Nature 308:32-36

Al-Amoudi A, Chang JJ, Leforestier A, McDowall A, Salamin LM, Norlén LP, Richter K, Blanc NS, Studer D, Dubochet J (2004a) Cryo-electron microscopy of vitreous sections. EMBO J 23:3583-3588

Al-Amoudi A, Norlén LP, Dubochet J (2004b) Cryo-electron microscopy of vitreous sections of native biological cells and tissues. J Struct Biol 148:131-135

Al-Amoudi A, Studer D, Dubochet J (2005) Cutting artifacts and cutting process in vitreous sections for cryo-electron microscopy. J Struct Biol 150:109-121

Bartesaghi A, Lecumberry F, Sapiro G, Subramaniam S (2012) Protein secondary structure determination by constrained single-particle cryo-electron tomography. Structure 20:2003-2013

Baumeister W, Steven AC (2000) Macromolecular electron microscopy in the era of structural genomics. Trends Biochem Sci 25:624-631

Beck M, Förster F, Ecke M, Plitzko JM, Melchior F, Gerisch G, Baumeister W, Medalia O (2004) Nuclear pore complex structure and dynamics revealed by cryo-electron tomography. Science 306:1387-1390

Bouchet-Marquis C, Hoenger A (2011) Cryo-electron tomography on vitrified sections: a critical analysis of benefits and limitations for structural cell biology. Micron 42:152-162

Bouchet-Marquis C, Pagratis M, Kirmse R, Hoenger A (2012) Metallothionein as a clonable high-density marker for cryoelectron microscopy. J Struct Biol 177:119-127

Braun W, Vasak M, Robbins AH, Stout CD, Wagner G, Kagi JH, Wuthrich K (1992) Comparison of the NMR solution structure and the x-ray crystal structure of rat metallothionein-2. Proc Natl Acad Sci USA 89:10124-10128

Briegel A, Dias DP, Li Z, Jensen RB, Frangakis AS, Jensen GJ (2006) Multiple large filament bundles observed in Caulobacter crescentus by electron cryotomography. Mol Microbiol 62:5-14

Briegel A, Ortega DR, Tocheva EI, Wuichet K, Li Z, Chen S, Müller A, Iancu CV, Murphy GE, Dobro MJ, Zhulin IB, Jensen GJ (2009) Universal architecture of bacterial chemoreceptor arrays. Proc Natl Acad Sci USA 106(40):17181-17186

Briggs JA (2013) Structural biology in situ-the potential of subtomogram averaging. Curr Opin Struct Biol 23:261-267

Campbell MG, Cheng A, Brilot AF, Moeller A, Lyumkis D, Veesler D, Pan J, Harrison SC, Potter CS, Carragher B, Grigorieff N (2012) 
Movies of ice-embedded particles enhance resolution in electron cryo-microscopy. Structure 20:1823-1828

Carlemalm E, Villiger W, Hobot JA, Acetarin JD, Kellenberger E (1985) Low temperature embedding with Lowicryl resins: two new formulations and some applications. J Microsc 140:55-63

Castano-Diez D, Al-Amoudi A, Glynn AM, Seybert A, Frangakis AS (2007) Fiducial-less alignment of cryo-sections. J Struct Biol 159: 413-423

Castano-Diez D, Scheffer M, Al-Amoudi A, Frangakis AS (2010) Alignator. A GPU-powered software package for robust fiducialless alignment of cryo tilt-series. J Struct Biol 170:117-126

Christensen AK (1971) Frozen thin sections of fresh tissue for electron microscopy, with a description of pancreas and liver. J Cell Biol 51: $772-804$

Cope J, Gilbert S, Rayment I, Mastronarde D, Hoenger A (2010) Cryoelectron tomography of microtubule-kinesin motor complexes. J Struct Biol 170:257-265

Cope J, Rank KC, Gilbert SP, Rayment I, Hoenger A (2013) Kar3Vik1 uses a minus-end directed Powerstroke for movement along microtubules. PLOS-one 8:e53792

Crowther RA, Amos LA, Finch JT, De Rosier DJ, Klug A (1970) Three dimensional reconstructions of spherical viruses by Fourier synthesis from electron micrographs. Nature 226:421-425

DeRosier DJ, Moore PB (1970) Reconstruction of three-dimensional images from electron micrographs of structures with helical symmetry. J Mol Biol 52:355-369

Diestra E, Fontana J, Guichard P, Marco S, Risco C (2009a) Visualization of proteins in intact cells with a clonable tag for electron microscopy. J Struct Biol 165:157-168

Diestra E, Cayrol B, Arluison V, Risco C (2009b) Cellular electron microscopy imaging reveals the localization of the Hfa protein close to the bacterial membrane. PLoS One 4(12):e8301

Dubochet J, Adrian M, Chang JJ, Homo JC, Lepault J, McDowall AW, Schultz P (1988) Cryo-electron microscopy of vitrified specimens. Q Rev Biophys 21:129-228

Dubochet J, Zuber B, Eltsov M, Bouchet-Marquis C, Al-Amoudi A, Livolant F (2007) How to "read" a vitreous section. Methods Cell Biol 79:385-406

Eibauer M, Hoffmann C, Plitzko JM, Baumeister W, Nickell S, Engelhardt $\mathrm{H}$ (2012) Unraveling the structure of membrane proteins in situ by transfer function corrected cryo-electron tomography. J Struct Biol 180:488-496

Feldheim DL, Eaton BE (2007) Selection of biomolecules capable of mediating the formation of nanocrystals. ACS Nano 1: $154-159$

Ferguson SM, Brasnjo G, Hayashi M, Wölfel M, Collesi C, Giovedi S, Raimondi A, Gong LW, Ariel P, Paradise S, O'Toole E, Flavell R, Cremona O, Miesenböck G, Ryan TA, De Camilli P (2007) A selective activity-dependent requirement for dynamin 1 in synaptic vesicle endocytosis. Science 316:570-574

Förster F, Pruggnaller S, Seybert A, Frangakis AS (2008) Classification of cryo-electron sub-tomograms using constrained correlation. J Struct Biol 161:276-286

Frank J (2009) Single-particle reconstruction of biological macromolecules in electron microscopy-30 years. Q Rev Biophys 42:139-158

Frank J, van Heel M (1982) Correspondence analysis of aligned images of biological particles. J Mol Biol 161:134-137

Frank GA, Bartesaghi A, Kuybeda O, Borgnia MJ, White TA, Sapiro G, Subramaniam S (2012) Computational separation of conformational heterogeneity using cryo-electron tomography and 3D sub-volume averaging. J Struct Biol 178:165-176

Gan L, Jensen GJ (2012) Electron tomography of cells. Q Rev Biophys $45: 27-56$

Goldie KN, Wedig T, Mitra A, Aebi U, Herrmann H, Hoenger A (2007) Dissecting the 3-D structure of vimentin intermediate filaments by cryo-electron tomography. J Struct Biol 158:378-385
Gonen T, Cheng Y, Sliz P, Hiroaki Y, Fujiyoshi Y, Harrison SC, Walz T (2005) Lipid-protein interactions in double-layered twodimensional AQP0 crystals. Nature 438:633-638

Gruska M, Medalia O, Baumeister W, Leis A (2008) Electron tomography of vitreous sections from cultured mammalian cells. J Struct Biol 161:384-392

Han HM, Zuber B, Dubochet J (2008) Compression and crevasses in vitreous sections under different cutting conditions. J Microsc 230: $167-171$

Henderson R, Baldwin JM, Ceska TA, Zemlin F, Beckmann E, Downing KH (1990) Model for the structure of bacteriorhodopsin based on high-resolution electron cryo-microscopy. J Mol Biol 213:899-929

Heumann JM, Hoenger A, Mastronarde DN (2011) Clustering and variance maps for cryo-electron tomography using wedge-masked differences. J Struct Biol 175:288-299

Hite RK, Raunser S, Walz T (2007) Revival of electron crystallography. Curr Opin Struct Biol 17:389-395

Höög JL, Schwartz C, Noon AT, O’Toole ET, Mastronarde DN, McIntosh JR, Antony C (2007) Organization of interphase microtubules in fission yeast analyzed by electron tomography. Dev Cell 12:349 361

Höög JL, Bouchet-Marquis C, McIntosh JR, Hoenger A, Gull K (2012) Cryo-electron tomography and 3-D analysis of the intact flagellum in Trypanosoma brucei. J Struct Biol 178:189-198

Hsieh CE, Marko M, Frank J, Mannella CA (2002) Electron tomographic analysis of frozen-hydrated tissue sections. J Struct Biol. 138(1-2): 63-73.

Jin L, Milazzo AC, Kleinfelder S, Li S, Leblanc P, Duttweiler F, Bouwer JC, Peltier ST, Ellisman MH, Xuong NH (2008) Applications of direct detection device in transmission electron microscopy. J Struct Biol 161:352-358

Kellenberger E (1991) The potential of cryofixation and freeze substitution: observations and theoretical considerations. J Microscopy 161: $183-203$

Kudryashev M, Stahlberg H, Castano-Diez D (2012) Limiting factors in single particle cryo electron tomography. Computational Struct Biotech J 1:1-6

Kühlbrandt W (1992) Two-dimensional crystallization of membrane proteins. Q Rev Biophys 25:1-49

Kuybeda O, Frank GA, Bartesaghi A, Borgnia M, Subramaniam S, Sapiro G (2013) A collaborative framework for 3D alignment and classification of heterogeneous sub-volumes in cryo-electron tomography. J Struct Biol 181:116-127

Li X, Mooney P, Zheng S, Booth CR, Braunfeld MB, Gubbens S, Agard DA, Cheng Y (2013) Electron counting and beam-induced motion correction enable near-atomic-resolution single-particle cryo-EM. Nat Methods 10:584-590

Liu H, Jin L, Koh SB, Atanasov I, Schein S, Wu L, Zhou ZH (2010) Atomic structure of human adenovirus by cryo-EM reveals interactions among protein networks. Science 329:1038-1043

Lucic V, Forster F, Baumeister W (2005) Structural studies by electron tomography: from cells to molecules. Annu Rev Biochem 74:833865

Lucic V, Rigort A, Baumeister W (2013) Cryo-electron tomography: the challenge of doing structural biology in situ. J Cell Biol 202:407419

Marsh BJ, Volkmann N, McIntosh JR, Howell KE (2004) Direct continuities between cisternae at different levels of the Golgi complex in glucose-stimulated mouse islet beta cells. Proc Natl Acad Sci U S A 101:5565-5570

Masich S, Ostberg T, Norlén L, Shupliakov O, Daneholt B (2006) A procedure to deposit fiducial markers on vitreous cryo-sections for cellular tomography. J Struct Biol 156:461-468

Mastronarde DN (2007) Fiducial marker and hybrid alignment methods for single- and double-axis tomography. In: Frank J (ed) Electron Tomography. Springer, New York, pp 163-185 
Mastronarde DN (2008) Correction for non-perpendicularity of beam and tilt axis in tomographic reconstructions with the IMOD package. $\mathrm{J}$ Microsc 230:212-217

Mastronarde DN, van der Heide PA, Morgan GP, Marsh BJ (2008) Supermontaging: reconstructing large cellular volumes by stitching together laterally adjacent tomograms. Microsc Microanal 14:106-107

McDonald K, Morphew MK (1993) Improved preservation of ultrastructure in difficult-to-fix organisms by high pressure freezing and freeze substitution: I. Drosophila melanogaster and Strongylocentrotus purpuratus embryos. Microsc Res Tech 24:465-473

McDowall AW, Chang JJ, Freeman R, Lepault J, Walter CA, Dubochet J (1983) Electron microscopy of frozen hydrated sections of vitreous ice and vitrified biological samples. J Microsc 131:1-9

McIntosh JR (2001) Electron microscopy of cells: a new beginning for a new century. J Cell Biol 153:F25-F32

McIntosh JR, Nicastro D, Mastronarde D (2005) New views of cells in 3D: an introduction to electron tomography. Trends Cell Biol 15:43-51

McMullan G, Clark AT, Turchetta R, Faruqi AR (2009a) Enhanced imaging in low dose electron microscopy using electron counting. Ultramicroscopy 109:1411-1416

McMullan G, Faruqi AR, Henderson R, Guerrini N, Turchetta R, Jacobs A, van Hoften G (2009b) Experimental observation of the improvement in MTF from backthinning a CMOS direct electron detector. Ultramicroscopy 109:1144-1147

Medalia O, Weber I, Frangakis AS, Nicastro D, Gerisch G, Baumeister W (2002) Macromolecular architecture in eukaryotic cells visualized by cryo-electron tomography. Science 298:1209-1213

Mercogliano CP, DeRosier DJ (2006) Gold nanocluster formation using metallothionein: mass spectrometry and electron microscopy. J Mol Biol 355:211-223

Miyazawa A, Fujiyoshi Y, Unwin N (2003) Structure and gating mechanism of the acetylcholine receptor pore. Nature 423:949-955

Nicastro D, Schwartz C, Pierson J, Gaudette R, Porter ME, McIntosh JR, 2006. The molecular architecture of axonemes revealed by cryoelectron tomography. Science 313:944-948

Nogales E, Wolf SG, Downing KH (1998) Structure of the alpha beta tubulin dimer by electron crystallography. Nature 391:199-203

O'Toole ET, Giddings TH Jr, Dutcher SK (2007) Understanding microtubule organizing centers by comparing mutant and wild-type structures with electron tomography. Methods Cell Biol 79:125-143

Patwardhan A, Carazo JM, Carragher B, Henderson R, Heymann JB, Hill E, Jensen GJ, Lagerstedt I, Lawson CL, Ludtke SJ, Mastronarde D, Moore WJ, Roseman A, Rosenthal P, Sorzano CO, Sanz-García E, Scheres SH, Subramaniam S, Westbrook J, Winn M, Swedlow JR, Kleywegt GJ (2012) Data management challenges in threedimensional EM. Nat Struct Mol Biol 19:1203-1207

Pelletier L, O’Toole ET, Schwager A, Hyman AA, Müller-Reichert T (2006) Centriole assembly in Caenorhabditis elegans. Nature 444: 619-623

Pierson J, Fernández JJ, Bos E, Amini S, Gnaegi H, Vos M, Bel B, Adolfsen F, Carrascosa JL, Peters PJ (2010) Improving the technique of vitreous cryo-sectioning for cryo-electron tomography: electrostatic charging for section attachment and implementation of an anti-contamination glove box. J Struct Biol 169:219-225

Pierson J, Ziese U, Sani M, Peters PJ (2011) Exploring vitreous cryosection-induced compression at the macromolecular level using electron cryo-tomography; $80 \mathrm{~S}$ yeast ribosomes appear unaffected. J Struct Biol 173:345-349

Risco C, Sanmartín-Conesa E, Tzeng WP, Frey TK, Seybold V, de Groot RJ (2012) Specific, sensitive, high-resolution detection of protein molecules in eukaryotic cells using metal-tagging transmission electron microscopy. Structure 20:759-766

Roy S, Olesiak M, Padar P, McCuen H, Caruthers MH (2012) Reduction of metal ions by boranephosphonate DNA. Org Biomol Chem 10: 9130-9133
Roy S, Olesiak M, Shang S, Caruthers MH (2013) Silver nanoassemblies constructed from boranephosphonate DNA. J Am Chem Soc 135: 6234-6241

Sader K, Studer D, Zuber B, Gnaegi H, Trinick J (2009) Preservation of high-resolution protein structure by cryo-electron microscopy of vitreous sections. Ultramicroscopy 110:43-47

Schur FK, Hagen WJ, de Marco A, Briggs JA (2013) Determination of protein structure at $8.5 \AA$ resolution using cryo-electron tomography and sub-tomogram averaging. J Struct Biol 184:394-400

Schwartz CL, Heumann JM, Dawson SC, Hoenger A (2012) A detailed, hierarchical study of Giardia lamblia's ventral disc reveals novel microtubule-associated protein complexes. PLoS One 7:e43783

Skiniotis G, Surrey T, Altmann S, Gross H, Song Y-H, Mandelkow E, Hoenger A (2003) Nucleotide-induced conformations in the neck region of dimeric kinesin. EMBO J 22:1518-1528

Slocik JM, Stone MO, Naik RR (2005) Synthesis of gold nanoparticles using multifunctional peptides. Small 1:1048-1052

Stanlis KK, McIntosh JR (2003) Single-strand DNA aptamers as probes for protein localization in cells. J Histochem Cytochem 51:797-808

Steven AC, Aebi U (2003) The next ice age: cryo-electron tomography of intact cells. Trends Cell Biol 13:107-110

Studer D, Michel M, Muller M (1989) High pressure freezing comes of age. Scanning Microsc 53:253-269

Taylor KA, Glaeser RM (1974) Electron diffraction of frozen, hydrated protein crystals. Science 186:1036-1037

Taylor KA, Glaeser RM (1976) Electron microscopy of frozen hydrated biological specimens. J Ultrastruct Res 55:448-456

Tokuyasu KT (1980) Immunochemistry on ultrathin frozen sections. Histochem J 12:381-403

Unwin PN, Henderson R (1975) Molecular structure determination by electron microscopy of unstained crystalline specimens. J Mol Biol 94:425-440

van Heel M, Frank J (1981) Use of multivariate statistics in analyzing the images of biological macromolecules. Ultramicroscopy 6:187-194

Veesler D, Campbell MG, Cheng A, Fu CY, Murez Z, Johnson JE, Potter CS, Carragher B (2013) Maximizing the potential of electron cryomicroscopy data collected using direct detectors. J Struct Biol 184:193-202

Wendt TG, Volkmann N, Goldie KN, Müller J, Mandelkow E, Hoenger A (2002) Microtubule binding patterns of the reverse kinesin motor Ncd reveal a minus-end directed power stroke. EMBO J 21:5969-5978

Werner JN, Chen EY, Guberman JM, Zippilli AR, Irgon JJ, Gitai Z (2009) Quantitative genome-scale analysis of protein localization in an asymmetric bacterium. Proc Natl Acad Sci U S A 106:7858-7863

Winkler H, Taylor KA (2006) Accurate marker-free alignment with simultaneous geometry determination and reconstruction of tilt series in electron tomography. Ultramicroscopy 106 : 240-254

Winkler H, Zhu P, Liu J, Ye F, Roux KH, Taylor KA (2009) Tomographic sub-volume alignment and sub-volume classification applied to myosin V and SIV envelope spikes. J Struct Biol 165:64-77

Xiong Q, Morphew MK, Schwartz CL, Hoenger A, Mastronarde DN (2009) CTF determination and correction for low dose tomographic tilt series. J Struct Biol 168:378-387

Yonekura K, Maki-Yonekura S, Namba K (2003) Complete atomic model of the bacterial flagellar filament by electron cryomicroscopy. Nature 424:643-650

Yu Z, Frangakis AS (2011) Classification of electron sub-tomograms with neural networks and its application to template-matching. J Struct Biol 174:494-504

Zanetti G, Riches JD, Fuller SD, Briggs JA (2009) Contrast transfer function correction applied to cryo-electron tomography and subtomogram averaging. J Struct Biol 168:305-312 